\title{
Acknowledgment to Reviewers
}

John R. Corboy, MD, FAAN, Editor; Laura B. Powers, MD, FAAN, Deputy Editor;

David C. Anderson, MD, FAAN, Associate Editor; Richard L. Barbano, MD, PhD, FAAN, Associate Editor

Sincere thanks to the individuals who have contributed to Neurology: Clinical Practice since it debuted in December 2011.

We welcome new reviewers. Please send an e-mail to ncpjournal@neurology.org if you have never reviewed for the journal but are interested in doing so. Please include a description of your credentials and expertise in the areas in which you are qualified to review.

The reviewers listed below with one asterisk have reviewed 5 or more articles. Two asterisks indicate that the reviewer has reviewed 10 or more manuscripts. This list includes those reviewers who returned a review or reviews of initial submissions (re-reviews of the same manuscript are not included) for the prior 5 years through December 1, 2015. Hereafter we will acknowledge our reviewers on an ongoing basis.

Jan O. Aasly

Lauren E. Abrey

Harold P. Adams

Gulsen Akman-Demir

Steven M. Albert

Michael P. Alexander

Enrique Alvarez

Maria Pia Amato

Catherine M. Amlie-Lefond

Beau M. Ances

Kaarin J. Anstey

Piero Antuono

Brian Stephen Appleby

Antonio Arjona

Carmel Armon

Melissa J. Armstrong

Isabelle Arnulf

Ethem Murat Arsava

Todd M. Arthur

Ganesh Asaithambi**

Rhoda Au

Sanford Auerbach

Alon Y. Avidan*

M. Ali Babi

David L. Bachman

Robert W. Baloh*

Philip Barber

Amit Bar-Or

A.M. Barrett

Kevin M. Barrett

J.D. Bartleson

Luca Bartolini

Joshua I. Barzilay
Rifaat M. Bashir

Alberto Battistini

Sebastian Bauer

Daniel Becker

Werner J. Becker

Selim R. Benbadis

Andreas Bender

Elinor Ben-Menachem

Michel Benoit

Roy G. Beran

José Berciano

Richard Beresford

Michel J. Berg

Donna C. Bergen

Joseph R. Berger*

Thomas Berger

Eric L. Berman

Gary L. Bernardini

James L. Bernat*

Tulio E. Bertorini

Daniel C. Bezerra

Erin D. Bigler

Jose Biller**

Damien Biotti

Frank Birklein

Alberto Bizzi

Andrew Bleasel

Thomas P. Bleck

Nicholas Alfred Blondin

Christopher J. Boes

Richard W. Bohannon

Roberto Bomprezzi

Soo Borson
Patrick Bosque

Teodoro Bottiglieri

Dennis Bourdette

James H. Bower

Allen Bowling

Allison Brashear

John C. Breitner

Bruce J. Brew

Amy Brodtmann*

Mark B. Bromberg

Matthijs C. Brouwer

Murray G. Brown

John C.M. Brust*

Jeffrey R. Buchhalter

Ferdinando S. Buonanno

Michelle A. Burack

Richard S. Burns

Cheryl D. Bushnell

Neil A. Busis*

Richard Byrne

Brian C. Callaghan

Laura Canafoglia

Louis R. Caplan*

Francisco Cardoso

Patrick Carney

Arturo Carpio

Jonathan Carr

Jonathan L. Carter

Richard J. Caselli

Barbara Casolla

Jose Luis Castillo

Pablo R. Castillo

Gastone G. Celesia 
Fernando Cendes

Marc C. Chamberlain*

Jane W. Chan

Ling Ling Chan

Seemant Chaturvedi**

Celia S. Chen*

Robert Chen

Shih-Pin Chen

Eric M. Cheng

Luisa Chiapparini

Jerome H. Chin

Daniel O. Claassen

Stacey Lynn Clardy

H. Brent Clark

David B. Clifford*

Adam Brian Cohen*

Bruce H. Cohen

Jeffrey A. Cohen

Ronald A. Cohen

James J. Corbett

David R. Cornblath

Bruce M. Coull

Michael B. Coulthart

Timothy J. Counihan

Antonio Culebras*

Marek Czosnyka

Russell C. Dale

Rohit Das

Martin Daumer

Gregory S. Day*

Roberto De Simone

Nicola De Stefano

Lisa M. DeAngelis

Anindita Deb

Ellen M. Deibert*

Bart M. Demaerschalk

Mariel Brooke Deutsch

Suhayl S. Dhib-Jalbut

Ellis R. Diamond

Dale Ding

Michael R. Dobbs

Bruce H. Dobkin

Michael J. Doherty

Peter D. Donofrio

E. Ray Dorsey*

Vanja C. Douglas

Barbara A. Dworetzky

George C. Ebers

Matthew A. Eccher

Jonathan C. Edwards

Mark J. Edwards

Ronald J. Ellis

Erik Fink Eriksen

Gail Eskes
Gregory Jacob Esper*

Patricio S. Espinosa

Mill Etienne

Amelia Evoli

Mustapha Ezzeddine

John Y. Fang

Constantine Farmakidis

Franz Fazekas

Dominic B. Fee

J. Americo M. Fernandes Jr

Eduardo Fernandez

Jose M. Ferro

Steven K. Feske

Terry D. Fife

Massimo Filippi

Christopher M. Filley

Howard Fillit

John N. Fink

Robert S. Fisher

Matthew L. Flaherty

Murray S. Flaster*

Bertrand Fontaine

Norman L. Foster

Robert J. Fox*

Gordon S. Francis

Samuel A. Frank

Gary M. Franklin

Mark S. Freedman

William D. Freeman

Jacqueline French

Robert P. Friedland

Deborah I. Friedman*

Joseph H. Friedman

Joseph Irwin Friedman

Jennifer E. Fugate

Kazuo Fujihara

Victor S.C. Fung

Ansgar J. Furst

Douglas Galasko

Steven Galetta*

Nicholas B. Galifianakis

Gloria Galloway

Gautam Ganguly

Erich W. Garland

Achim Gass

Thomas Gasser

John Gaughen

Jeroen J.G. Geurts

Sarah Gheuens*

Barry E. Gidal

Gordon J. Gilbert

Don Gilden*

Wendy Gilmore

Elias A. Giraldo**
Eli Glatstein

Thomas H. Glick*

Peter J. Goadsby

Stefan Martin Golaszewski

Jill S. Goldman

Myla D. Goldman

Serge Goldman

Larry B. Goldstein*

Clifton L. Gooch

Philip B. Gorelick

Mark J. Gorman

Kenneth C. Gorson

Rebecca F. Gottesman

Raghav Govindarajan

Jerome J. Graber

Neill R. Graff-Radford

Benjamin M. Greenberg

James Greene

Wolfgang Grisold

Gary Gronseth

Michael Gruenthal**

Jeff Guptill

M. Edip Gurol

Mary N. Haan

Marios Hadjivassiliou

Brian Hainline

Gabor Michael Halmagyi*

John J. Halperin

Judith U. Harrer*

Taylor Harrison

Brian C. Healy

Sandra L. Helmers

Thomas M. Hemmen

Clare Henchcliffe

J. Craig Henry

Thomas R. Henry

John W. Henson

Michael D. Hill

Daniel B. Hoch

Sara E. Hocker

Charles Hodge

John R. Hodges

Anna DePold Hohler*

Reinhard Hohlfeld

Robert G. Holloway*

Justin M. Honce

Adilia Hormigo

Jonathan P. Hosey

Sung-Tsang Hsieh

Jason Haitao Huang

R.A.C. Hughes

Thierry Huisman

Thomas Hummel

Peter Huppke 
Haitham M. Hussein**

John C. Hutchins

Michael Hutchinson

David Y. Hwang

Kachi Illoh

Sarosh R. Irani

Giris Jacob

Bart C. Jacobs

Daniel H. Jacobs

Teresa L. Jacobs

Claudia Jacova

William J. Jagust

Babak S. Jahromi

Todd Janus

Edward C. Jauch

Annapurni V. Jayam-Trouth

Rou-Chen Jee

Lara E. Jehi

Nathalie Jette

Glen Jickling

Xinchun Jin

Keith A. Johnson

Laurentiu Jomir

Elaine C. Jones

Lyell K. Jones

Ralph F. Jozefowicz

Larry Junck*

Michael T. Jurkiewicz

Norman J. Kachuck

Kai Kallenberg

Lalit Kalra

Keiko Kamakura

Carlos Kamiya-Matsuoka

Amit Kansara

Tulay Kansu

Orhun H. Kantarci

Gregory Kapinos*

Jaideep Kapur

Matthias Karst

Jorge C. Kattah**

Lars Kellert

Brendan J. Kelley

Gordon R. Kelley

Vladimir Kepe

Kevin A. Kerber

Yasmin Khakoo

Jaffar Khan

Tarannum S. Khan

Priyank Khandelwal

Ronald J. Killiany

Laurence J. Kinsella

Douglas B. Kirsch

Howard S. Kirshner

Ilya Kister
Autumn Marie Klein

Pavel Klein

Timothy John Kleinig

Andreas E. Koch

Sebastian Koch

Matthew A. Koenig

Maja Kojovic

Katie Kompoliti

Barbara S. Koppel**

Suresh Kotagal

Karim Hussein Kotkata

Lauren B. Krupp

Wilhelm Kuker

Elena Kuklina

Roger W. Kula

Neeraj Kumar

Jeffrey S. Kutcher

Gert Kwakkel

Susan Landau

Basile Nicolas Landis

Bethan Lang

Frederick Langendorf

John T. Langfitt

Douglas J. Lanska

Dan Larriviere

Julius Gene Silva Latorre

Robert Laureno

Susan Law

Victoria H. Lawson

Ronald M. Lazar

Kiwon Lee

Stephane Legriel

Maria Isabel S. Leite

Ronald P. Lesser

Steven R. Levine

Steven L. Lewis

Frank Leypoldt

Didier Leys

Jane L. Liesveld

David J. Likosky

Chih-Chun Lin

Geoffrey S.F. Ling

Kore Liow

Rajka M. Liscic

Jerome P. Lisk*

Warren D. Lo

Eric L. Logigian

Giancarlo Logroscino

Carla F. LoPinto-Khoury

Dana Lustbader

Robert A. Lustig

John Lynch

Timothy Lynch

Edward Maa
Paul J. Maccabee

Kenneth J. Mack

Philippe Maeder

Melinda Magyari

Atul Maheshwari

Mark W. Mahowald

Michael Makdissi

C.J. Malanga

Paresh A. Malhotra

David E. Mandelbaum

Raffaele Manni

Mark E. Mapstone

Jonathan Andrew

Marcus

John D. Markman

Ruth Ann Marrie

James J. Marriott

Wayne Martin

Catherine Masson

Jim A. Mastrianni

David B. Matchar

Makoto Matsui

Alberto Maud*

Anna Teresa Mazzeo

Paolo Aurelio Maria

Mazzone

Gary N. Mcabee

Justin C. McArthur

Micheline McCarthy

Andrew McKeon

Michael P. McQuillen

Kimford J. Meador

Ravi Menon

Matthew N. Meriggioli

Jose Guillermo Merino*

Steven R. Messe

Albee Messing

Ana-Claire L. Meyer

Jamal A. Mikdashi

Bradford Miller

Daniel Miller

John W. Miller

Neil R. Miller

Scott Mintzer

Nishant K. Mishra

Panayiotis D. Mitsias

Manoj K. Mittal

Heidi Moawad**

Jennifer Molano

Francesca Morgante

A. Jenny Morton

Thierry Moulin

Maciej M. Mrugala

Susanne Muehlschlegel 
Keith W. Muir

Marco Mula

Gary J. Myers

Georges Naassaan

Andrew M. Naidech

Seema Nair

Pushpa Narayanaswami

Avindra Nath**

Paul J. Nederkoorn

Maromi Nei

Mary Elizabeth Nelson

Jonathan Newmark

John Ney**

Yu-tze Ng

Fenwick T. Nichols

Rachel Nolan

Andrew Norden

Marc R. Nuwer**

Obioma Nwaiwu

Paul W O'Connor

Shin J. Oh

Darin T. Okuda

Michael S. Okun

Stephen M. Oppenheimer

Richard Orrell

Michael O’Sullivan

Brian R. Ott

Leonardo Pantoni

Jessica Panzer

Eleftherios S. Papathanasiou

Alvaro Pascual-Leone

Nitin C. Patel

Gary L. Pattee

Friedemann Paul

B. Lee Peterlin

Thanh G. Phan

Lara Pilutti

Sean J. Pittock

Michael Polydefkis

Aurel Popa-Wagner

Eric E. Potts

William J. Powers

John C. Probasco**

Amy A. Pruitt*

Mary Jo V. Pugh

Patrick M. Pullicino

Jukka Putaala

Carlo Cosimo Quattrocchi

Aldo Quattrone

Gil Dan Rabinovici

Alejandro A. Rabinstein

Mubeen F. Rafay

Jeffrey J. Raizer

Vedantam Rajshekhar
Michael Rasminsky

Marcia Hillary Ratner

Gerald V. Raymond

Anthony T. Reder

Stephen G. Reich

Stephen C. Reingold

Awais Riaz

Irene H. Richard

Jack E. Riggs

Steven P. Ringel

E. Bernd Ringelstein

Richard Alan Rison

Martin A. Ritter

Michael H. Rivner

E. Steve Roach

Matthew Stuart Robbins

Gustavo J. Rodriguez*

Lisa R. Rogers

Terry Rolan**

Michael Ronthal

Karen L. Roos

Myrna R. Rosenfeld

David B. Rosenfield

Felix Rosenow

David A. Rottenberg

Andrew S. Rowland

Lewis P. Rowland

Todd Rowland

Emile P. Roy

Walter Royal III

Janet Rucker

Stephan J. Rüegg*

Rosanna Sabini

Tonia Marie Sabo

Tiina Sairanen

Johnny S. Salameh

Michael Martin Saling

Antonio J. Sanchez

Friedhelm Sandbrink

Veronica Elisa Santini

Maher Saqqur

Pratheesh Sathyan

Saty Satya-Murti**

Laura Scherer

Wouter Ingmar Schievink

David Schiff

Erich Schmutzhard

Raphael Schneider

Ruth Schneider

Susanne A. Schneider

Menno M. Schoonheim

Nina F. Schor

Matthew Schrag

GianPietro Sechi
Raymond C.S. Seet

Souvik Sen

Alessandro Serra

Aasef G. Shaikh

Vijay K. Sharma

Sunil A. Sheth

Michael E. Shy

Bruce Sigsbee

Mauro Silvestrini

Jack H. Simon

Neil G. Simon

David M. Simpson

Harvey S. Singer

Samuel Singer

Anuradha Singh

Sanjay P. Singh

Nilika Shah Singhal

Saurabh R. Sinha

Aksel Siva

Chris Skedgel

A. Gordon Smith

Jonathan K. Smith

Salil Soman

Claudia Sommer

Sarah Song

Marianna V. Spanaki

Roderick Spears

Luciano A. Sposato

Christian Stapf

Robert M. Starke

Israel Steiner

Barney J. Stern

James C. Stevens

Jon Stone

Michael Strupp

Adnan Subei

Prem S. Subramanian

Campbell Sullivan

James F. Sumowski

Jerzy P. Szaflarski

Viktor Szeder

David Tanne

Turgut Tatlisumak

Jinny Tavee

Lynne P. Taylor

Olli Tenovuo

Richard B. Tenser

Alan J. Thompson

Matthew James Thurtell

David Tirschwell

Maarten J. Titulaer

David C. Tong*

Diego R. Torres-Russotto

Anthony L. Traboulsee 
Bryan J. Traynor William J. Triggs Manjari Tripathi Jack W. Tsao* John Tulloch Hayrettin Tumani Tanya N. Turan Kenneth L. Tyler* William R. Tyor Marcus M. Unger Basim M. Uthman Maarten Uyttenboogaart Josep Valls-Sole Laura van de Pol Ludo W. van der Pol Gregory P. Van Stavern Joe Verghese

Thomas Vidic

Pablo Villoslada

Angela Vincent Timothy L. Vollmer Raymond D. Voltz Annabel Kim Wang
Leo H. Wang

Alex Ward

Katja E. Wartenberg

Allison L. Weathers

Donald F. Weaver

Lawrence R. Wechsler

Louis H. Weimer

Brian G. Weinshenker

Dan Weisholtz

Howard D. Weiss

John R. Wherrett

Roberta F. White

Will N. Whiteley

Gerald J. Wiest

Eelco F.M. Wijdicks

Tissa Wijeratne*

Korwyn Williams

Michael A. Williams

Andrew N. Wilner

Jeffrey L. Winters

Eric T. Wong

Hal Wortzel

Thomas Wychowski
Li Xiong

Junqian Xu

Hiroshi Yamagami

Paul A. Yates

E. Ann Yeh*

Tomokatsu Yoshida

G. Bryan Young

Kate C. Young

Robert J. Young

William B. Young

Michael E. Yurcheshen

Osama O. Zaidat

Joanna M. Zakrzewska

Edward Zamrini

Michael S. Zandi

Inga Zerr

Qiuhua Zhang

Xing-Quan Zhao

Sasa Zivkovic

Douglas W. Zochodne

Alexander Y. Zubkov

Jose Rafael Pantoja

Zuzuarregui

\section{DISCLOSURES}

John R. Corboy, MD, FAAN, conducts research supported by the NIH, PCORI, Novartis, Sun Pharma, the National Multiple Sclerosis Society, NMSS (MS Tissue Bank), and Diogenix and has been a consultant for Genentech, Novartis, Teva Neurosciences, and Biogen Idec. Dr. Corboy has received honoraria for speaking from AAN, PRIME Education, Inc., and the Rocky Mountain MS Center. He is a Board Member of the NMSS Colorado-Wyoming chapter, and receives an honorarium from the AAN as an Associate Editor of Neurology and Editor of Neurology: Clinical Practice. Laura B. Powers, MD, FAAN, has received speaker honoraria from AAN and state neurological societies, and serves as Associate Editor for Coding for Continuum and as Deputy Editor of Neurology: Clinical Practice. David C. Anderson, MD, FAAN, has served on event adjudication committees for NIH (NHLBI, NINDS), serves on data safety and monitoring boards for NIH/ NICHD and Bayer, and as co-investigator and co-PI for NIH/NINDS. Dr. Anderson serves as associate editor of Neurology: Clinical Practice and associate editor of Continuum Audio. Richard L. Barbano, MD, PhD, FAAN, serves on a scientific advisory board for Allergan, serves as an associate editor for Neurology: Clinical Practice, performs botulinum toxin injections at the University of Rochester (30\% effort); receives research support from Allergan, Vaccinex, and Biotie; has received research support from NIH (NINDS, ORDR): Dystonia Coalition Projects, Site PI; holds stock/stock options in VisualDx; and has served as an expert witness in legal proceedings including malpractice, not involving commercial entities.

\section{What's New in Clinical Practice?}

Neurology ${ }^{\circledR}$ Clinical Practice now has podcasts available for download. The podcasts are introduced by Editor John R. Corboy, MD, FAAN, who highlights papers from the current issue. The interview that follows features authors discussing papers in more depth and bringing clinical implications to the fore. 


\section{Neurology ${ }^{\circ}$ Clinical Practice}

Acknowledgment to Reviewers

John R. Corboy, Laura B. Powers, David C. Anderson, et al.

Neurol Clin Pract 2016;6;2-6

DOI 10.1212/CPJ.0000000000000224

This information is current as of February 15, 2016

Updated Information \&

Services

Permissions \& Licensing

Reprints including high resolution figures, can be found at:

http://cp.neurology.org/content/6/1/2.full.html

Information about reproducing this article in parts (figures,tables) or in its entirety can be found online at:

http://cp.neurology.org/misc/about.xhtml\#permissions

Information about ordering reprints can be found online:

http://cp.neurology.org/misc/addir.xhtml\#reprintsus

Neurol Clin Pract is an official journal of the American Academy of Neurology. Published continuously since 2011, it is now a bimonthly with 6 issues per year. Copyright (C) 2016 American Academy of Neurology. All rights reserved. Print ISSN: 2163-0402. Online ISSN: 2163-0933.

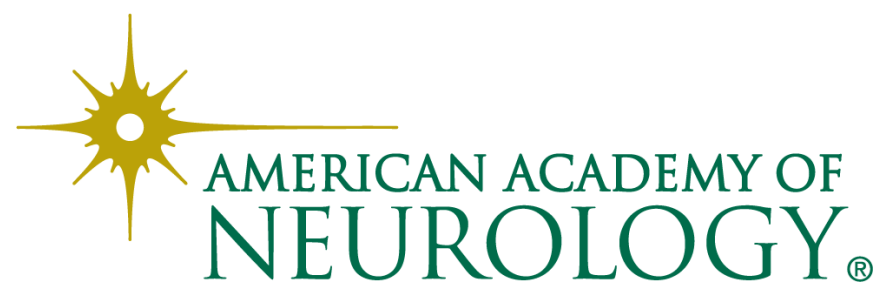

